\title{
Impulse response measurement using colored pseudo noise with low crest factor
}

\author{
Ryo Shinohara and Yutaka Kaneda* \\ Graduate School of Engineering, Tokyo Denki University, \\ 5 Senzyu-Asahi-cho, Chiyoda-ku, Tokyo,120-8551 Japan
}

(Received 20 May 2014, Accepted for publication 12 June 2014)

Keywords: Impulse response measurement, Colored pseudo noise, Crest factor reduction, Minimum noise PACS number: 43.58.-e, 43.58.Gn, 43.60.-c [doi:10.1250/ast.35.321]

\section{Introduction}

Impulse response has been widely used to measure the frequency characteristics and reverberation time of acoustic systems, and its measurement is thus essential. We have proposed minimum-noise swept sine (MN-SS) signal that can minimize the noise power included in measurement results and verified the validity of the MN-SS signal [1].

In addition to the swept sine (SS) signal, pseudo-noise (PN) signal can be used as the measurement signal. PN signals are advantageous because they are less affected by nonstationary noise than SS signals. However, PN signals have a high crest factor, or peak-to-root mean square (RMS) ratio, leading to a low signal-to-noise $(\mathrm{SN})$ ratio of measurement results.

A method for reducing the crest factor of the PN signal was proposed by Ouderaa et al. [2]. However, because the method performs clipping to a digital signal, the maximum amplitude is not reduced enough after the signal is converted to an analog signal.

This paper proposes an effective crest factor reduction method that interpolates a digital signal for getting close to an analog signal before clipping. Then, we apply it to minimumnoise PN (MN-PN) signals that can minimize the noise power included in measurement result, to confirm the validity of the proposed method.

\section{Colored pseudo noise}

The power level of noise included in the measured impulse response depends on the power spectrum $P_{\mathrm{S}}(\omega)$ of the measurement signal. Therefore, it is important to use a measurement signal with an appropriate power spectrum $P_{\mathrm{S}}(\omega)$. The spectrum $S(\omega)$ of colored PN having a power spectrum $P_{\mathrm{S}}(\omega)$ is expressed as

$$
S(\omega)=\sqrt{P_{\mathrm{S}}(\omega)} \cdot \exp (j \theta(\omega)),
$$

where the phase spectrum $\theta(\omega)$ is given as a random number. Time signal is obtained by the inverse Fourier transform of $S(\omega)$.

\section{Method of crest factor reduction}

\subsection{Crest factor of digital signal}

The crest factor of a signal is defined as the ratio of its maximum absolute value of amplitude to its RMS value.

\footnotetext{
*e-mail: kaneda@c.dendai.ac.jp
}

Because a digital signal is subjected to time-axis interpolation during conversion to an analog signal, the maximum amplitude is increased as shown in Fig. 1.

Therefore, when the crest factor of the analog signal which is used in an actual environment is calculated using a digital signal, it is necessary to predict the maximum amplitude of the analog signal after interpolation and calculate the crest factor. Thus, for example, the crest factor of a digital signal consisting of only 1 and -1 , such as an maximum length sequence (MLS), takes high value, approximately 2.7 , instead of 1 , as an analog signal.

3.2. Crest factor reduction method

Figure 2 shows the proposed method of reducing the crest factor which is developed on the basis of the method proposed by Ouderaa et al.

(a) The amplitude of the original signal (colored PN) is clipped after interpolation, which leads to a reduction in the maximum amplitude and a crest factor reduction.

(b) However, the signal power spectrum changes owing to clipping. To solve this problem, the signal is decimated by the original sampling rate and subjected to discrete Fourier transform (DFT), then, the power spectrum of the obtained signal is corrected to that of the original signal.

(c) The crest factor of the signal derived by applying inverse DFT to the spectrum obtained in step $(b)$ is calculated to determine the convergence of the crest factor. If the convergence is unsatisfactory, the above steps are iterated.

By iterating the steps of operation as explained above repeatedly, a signal with a power spectrum identical to that of the original signal and a reduced crest factor can be obtained.

3.3. Confirmation of the effectiveness of the proposed method

The difference between the proposed method and the conventional one [2] lies in that the proposed method takes into account the changes in the maximum amplitude caused by interpolation. Using the colored $\mathrm{PN}$ having $1 / f$ spectrum, the convergence characteristics of the algorithms of the proposed and the conventional methods were compared. Interpolation ratio in the proposed method was set to be 8 .

The clipping level of the conventional method was set to be $95 \%$ of the maximum amplitude as stated in the literature of Ouderaa et al. The clipping level of the proposed method was set to be 1.15 times as much as the RMS value. Figure 3 


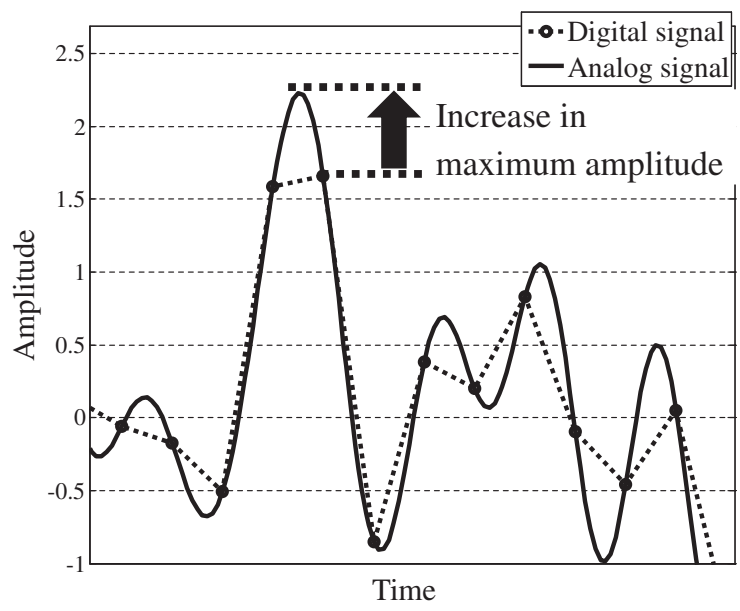

Fig. 1 Change in maximum amplitude by interpolation.

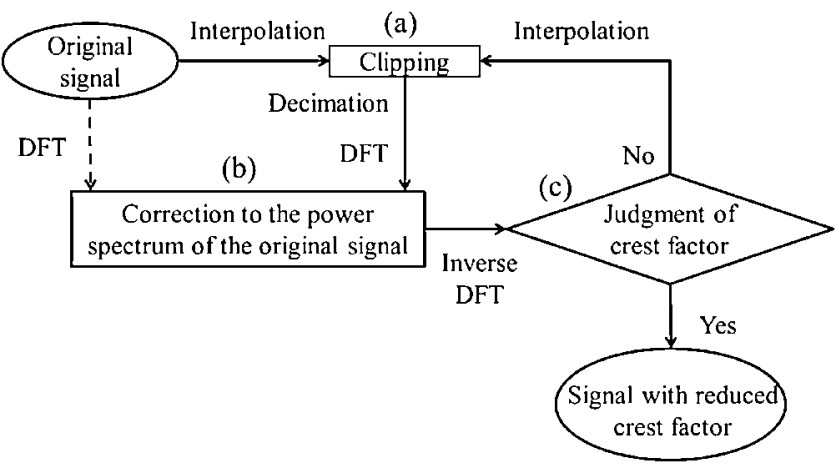

Fig. 2 Proposed algorithm for crest factor reduction.

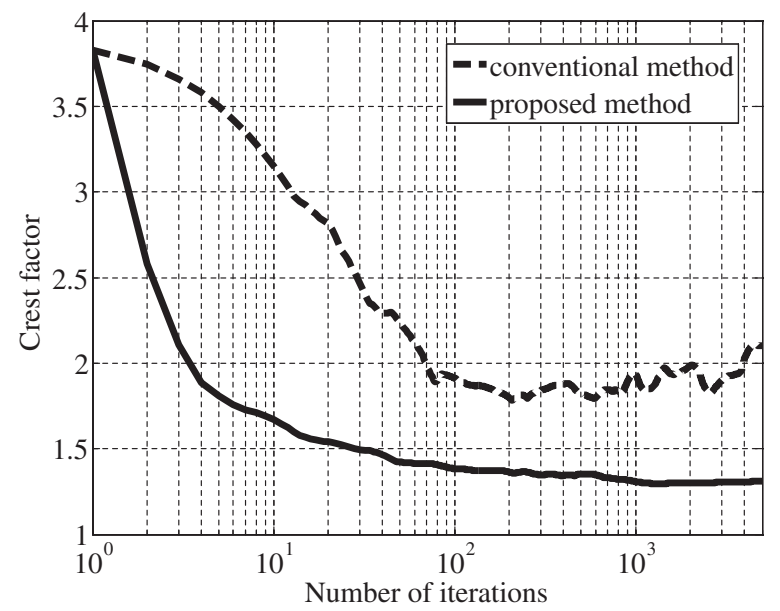

Fig. 3 Convergence characteristics of crest factor of conventional and proposed method.

shows the convergence characteristics of crest factor of the two methods for up to 5,000 iterations. The minimum crest factors of the conventional and proposed methods are 1.81 and 1.28, respectively. This demonstrates that the crest factor improvement of about $3 \mathrm{~dB}$ is obtained by applying the proposed method.
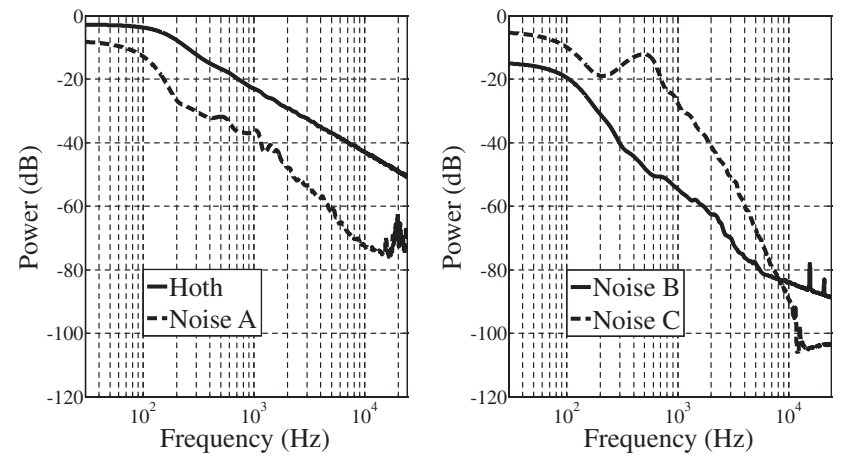

Fig. 4 Spectra of four noises.
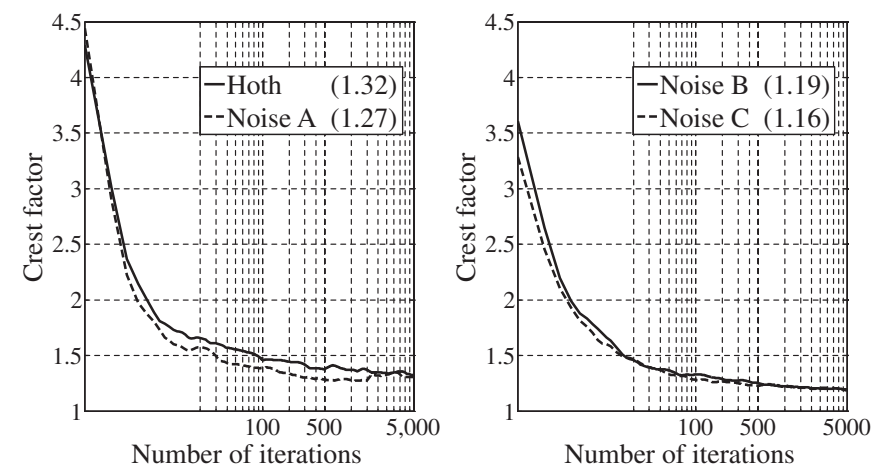

Fig. 5 Convergence characteristics of crest factor reduction algorithm.

\section{Impulse response measurement simulation}

\subsection{Conditions for simulation}

To confirm the noise reduction effect of the MN-PN signal with a reduced crest factor, the measurement of impulse response under noisy condition was simulated. The spectra of the four types of noise used in the simulation are shown in Fig. 4. An impulse response of a loudspeaker measured in an anechoic room was used as that of an unknown system.

As measurement signals, three types of signal, i.e., the MN-SS signal and the MN-PN signals before (original) and after reducing the crest factor, were used. The signal length was set to be $2^{15}$, and the maximum amplitude of the three signals were adjusted to be identical. The crest factor of the MN-SS signal was calculated to be about 1.7.

4.2. Synthesis of MN-PN signal with reduced crest factor

The power spectrum $P_{\mathrm{S}}(\omega)$ of the measurement signal that minimizes the noise power contained in measurement results in given by [1]

$$
P_{\mathrm{S}}(\omega)=C \cdot \sqrt{P_{\mathrm{N}}(\omega)} \quad C: \text { Constant, }
$$

where $P_{\mathrm{N}}(\omega)$ is the power spectrum of stationary ambient noise. By substituting the equation (2) into (1) the original MN-PN signal was synthesized, and its crest factor was reduced by the algorithm show in Fig. 2 .

4.3. Simulation result

Figure 5 shows the convergence characteristics of the crest factor reduction algorithm for each noise, controlling the clipping level to 1.15 times the RMS value. The minimum 

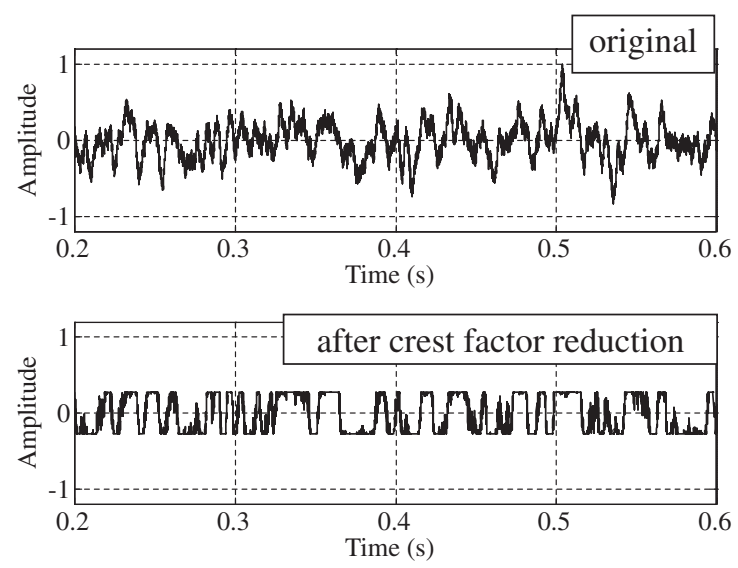

Fig. 6 MN-PN waveforms before and after crest factor reduction.

crest factors obtained in 5000 iteration are shown in the upper right parentheses. For any noise, the minimum crest factor is less than $\sqrt{2}=1.41$, which is the crest factor of a sine wave.

Figure 6 shows examples of the waveform of MN-PN signals before (original) and after the crest factor reduction. They have the same spectrum and RMS value, but the maximum amplitude is reduced after the crest factor reduction.

Table 1 shows a summary of the noise level included in the impulse response measured using the MN-SS signal and the MN-PN signal before (original) and after the crest factor reduction. The numerical values in the table are presented in $\mathrm{dB}$ relative to the noise level obtained using an SS signal having a white spectrum [3].

As shown in Table 1, the noise level for the MN-PN signal with a reduced crest factor is $14-24 \mathrm{~dB}$ lower than that for the SS signal with a white spectrum. This is due to the noise minimum spectrum shown by Eq. (2). The MN-PN signal with a reduced crest factor was found to have a higher
Table 1 Noise level of impulse response obtained using the MN-SS and MN-PN signals.

\begin{tabular}{ccccc}
\hline & Hoth & Noise A & Noise B & Noise C \\
\hline MN-SS & $-10.9 \mathrm{~dB}$ & -19.7 & -20.3 & -15.0 \\
\hline $\begin{array}{c}\text { MN-PN } \\
\text { original }\end{array}$ & -4.7 & -13.3 & -14.7 & -9.1 \\
\hline $\begin{array}{c}\text { MN-PN } \\
\text { after crest factor } \\
\text { reduction }\end{array}$ & -14.2 & -22.7 & -24.2 & -18.2 \\
\hline
\end{tabular}

noise-reducing effect by approximately $9 \mathrm{~dB}$ and $3 \mathrm{~dB}$ than those of the original MN-PN signal and the MN-SS signal, respectively.

\section{Conclusion}

In this study, we proposed a new algorithm for reducing the crest factor of the pseudo noise, and verified its effectiveness. Using the new algorithm, we reduced the crest factor of the colored MN-PN signal that minimizes the noise power included in the measured impulse response. The crest factor of the MN-PN signal after reduction was lower than that of the MN-SS signal, and a higher noise-reducing effect was confirmed.

\section{References}

[1] N. Moriya and Y. Kaneda, "Impulse response measurement that maximizes signal-to-noise ratio against ambient noise," Acoust. Sci. \& Tech., 28, 43-45 (2007).

[2] E. V. Ouderaa, J. Schoukens and J. Renneboog, "Peak factor minimization using a time-frequency domain swapping algorithm," IEEE Trans. Instrum. Meas., 37, 145-147 (1988).

[3] Y. Suzuki, F. Asano, H. Kim and T. Sone, "An optimum computer-generated pulse signal suitable for the measurement of very long impulse responses," J. Acoust. Soc. Am., 97, 11191123 (1995). 